\title{
Author Correction: Faster, sharper, and deeper: structured illumination microscopy for biological imaging
}

Yicong Wu (D) and Hari Shroff

Correction to: Nature Methods https://doi.org/10.1038/s41592-018-0211-z, published online 26 November 2018

In the version of this Perspective originally published, Fig. $4 \mathrm{~g}$ included an incorrect inset adapted from a different figure than the main image in the panel. This error has been corrected in the PDF and HTML versions of the paper.

\section{Author Correction: Genome-wide SWAp-Tag yeast libraries for proteome exploration \\ Uri Weill, Ido Yofe, Ehud Sass, Bram Stynen, Dan Davidi, Janani Natarajan, Reut Ben-Menachem, Zohar Avihou, Omer Goldman, Nofar Harpaz, Silvia Chuartzman, Kiril Kniazev, Barbara Knoblach, Janina Laborenz, Felix Boos, Jacqueline Kowarzyk, Shifra Ben-Dor (D), Einat Zalckvar, Johannes M. Herrmann, Richard A. Rachubinski (D), Ophry Pines, Doron Rapaport, Stephen W. Michnick, Emmanuel D. Levy $\mathbb{D}$ and Maya Schuldiner (D)}

Correction to: Nature Methods https://doi.org/10.1038/s41592-018-0044-9, published online 9 July 2018

The version of Supplementary Table 1 originally published online with this article contained incorrect localization annotations for one plate. This error has been corrected in the online Supplementary Information.

\section{Author Correction: A pH-correctable, DNA-based fluorescent reporter for organellar calcium}

Nagarjun Narayanaswamy (D), Kasturi Chakraborty (D), Anand Saminathan, Elizabeth Zeichner, KaHo Leung (iD, John Devany and Yamuna Krishnan (D)

Correction to: Nature Methods https://doi.org/10.1038/s41592-018-0232-7, published online 10 December 2018

The originally published paper has been updated to include the following new reference, added as ref. 18: Albrecht, T., Zhao, Y., Nguyen, T. H., Campbell, R. E. \& Johnson, J. D. Fluorescent biosensors illuminate calcium levels within defined beta-cell endosome subpopulations. Cell Calcium 57, 263-274 (2015). Subsequent references have been renumbered in the reference list and throughout the text. Minor text changes were made in the sentence in which this new reference is first cited: "Previous attempts used endocytic tracers bearing either $\mathrm{pH}$ - or $\mathrm{Ca}^{2+}$-sensitive dyes to serially measure population-averaged $\mathrm{pH}$ and apparent $\mathrm{Ca}^{2+}$ in different batches of cells, thus scrambling information from individual endosomes ${ }^{13-17 "}$ in the original introduction was changed to "Previous attempts used endocytic tracers bearing either $\mathrm{pH}$ - or $\mathrm{Ca}^{2+}$-sensitive dyes $\mathrm{s}^{13-17}$ or fluorescent-protein-based sensors ${ }^{18}$ to serially measure population-averaged $\mathrm{pH}$ and apparent $\mathrm{Ca}^{2+}$ in different batches of cells, thus scrambling information from individual endosomes." These changes have been made in the HTML and PDF versions of the article. 